\title{
Network Formation for Asymmetric Players and Bilateral Contracting*
}

\author{
Carme Àlvarez Maria Serna Aleix Fernàndez \\ ALBCOM Research Group. \\ Computer Science Department. \\ Universitat Politècnica de Catalunya, Barcelona TECH.
}

\begin{abstract}
We study a network formation game where players wish to send traffic to other players. Players can be seen as nodes of an undirected graph whose edges are defined by contracts between the corresponding players. Each player can contract bilaterally with others to form bidirectional links or break unilaterally contracts to eliminate the corresponding links. Our model is an extension of the traffic routing model considered in $[5,6,7]$ in which we do not require the traffic to be uniform and all-to-all. Player $i$ specifies the amount of traffic $t_{i j} \geq 0$ that wants to send to player $j$. Our notion of stability is the network pairwise Nash stability, when no node wishes to deviate unilaterally and no pair of nodes can obtain benefit from deviating bilaterally. We show a characterization of the topologies that are pairwise Nash stable for a given traffic matrix. We prove that the best response problem is NP-hard and devise a myopic dynamics so that the deviation of the active node can be computed in polynomial time. We show the convergence of the dynamics to pairwise Nash configurations, when the contracting functions are anti-symmetric and affine, and that the expected convergence time is polynomial in the number of nodes when the node activation process is uniform.
\end{abstract}

keywords network formation games bilateral contracting pairwise Nash equilibrium myopic dynamics

\section{Introduction}

Nowadays social and economic networks, and even communication networks are typically endogenous and operate at a scale that makes unpractical the use

*work partially supported by funds from the Spanish Ministry for Economy and Competitiveness (MINECO) and the European Union (FEDER funds) under grant TIN201346181-C02-1-R (COMMAS) and from AGAUR, Generalitat de Catalunya, under grant SGR 2014:1137 (ALBCOM). 
of centralized policies. In all these networks, nodes can be seen as autonomous agents that wish to communicate to each other. Each agent may choose whom to accept connections from or whom to connect to. Moreover nodes can also decide to break a set of non profitable connections. A general framework for the study of network creation with selfish agents was proposed by Jackson and Wolinsky [17]. Different extensions to this model are numerous in Economics (see the survey [16]). Our study focuses on network formation games for modeling the formation of communication networks. Fabrikant et al. [15] introduce a novel game that models the creation of Internet-like networks by selfish node-agents without central coordination. Nodes pay for links that they establish unilaterally and benefit from short paths to all destinations. Hence, the cost of each node has two components: the total cost of the edges created by this node plus the sum of distances from the node to all the others. The authors assume that all pairs of nodes have the same interest (all-to-all communication pattern with identical weights), the cost of being disconnected is infinite and the edges paid by one node can be used by others. They do not look at the dynamics for network creation in which networks are developed in stages where nodes can add or delete links at each stage. Albers et al. in [1,2] continue the study of the model of [15] improving some of their bounds of the price of anarchy.

Corbo and Parkes [10] generalize the model of Fabrikant et al. [15] by having links formed bilaterally instead of unilaterally and having the cost of links shared equally between both end nodes. In subsequent works many other different network formation games have been defined by considering distinct cost functions or different kind of strategies jointly with their appropriate equilibrium concept (see $[11,19,9,13,12,4,3,18,8,14]$ ).

Some common characteristics of all the previously referred models are that each pair of nodes want to communicate to each other and that the cost function depends on the distance to other nodes among other components. In the models studied by Arcaute el al. in $[5,6,7]$ each node derives utility from connectivity and incurs a cost. The cost is comprised of three different terms: the cost of routing traffic, the maintenance cost of its links, and the disconnection cost. The routing cost depends on the amount of traffic routed through the node instead of the distances to all the other nodes. The player's utility comes from the payments for the contracts which are a natural mechanism to compensate the costs of creating links. In this model link formation is bilateral, while link elimination is unilateral, and then an appropriate notion of stability is captured by Pairwise Nash equilibrium, as it is argued in $[5,7]$. The authors study such game under a form of myopic best response dynamics and they characterize a set of assumptions under which these dynamics converges to a stable network. The authors left as an open question the extension of the model defined in $[5,7]$ to asymmetric traffic matrix instead of the uniform all-to-all traffic considered in the paper. They also suggest to expand the strategy space considered by each node in their dynamics.

In this work we extend the model of Arcaute et al. [5, 7] by considering asymmetric players. Each player $i$ specifies the amount of traffic $t_{i j}$ that desires to send to player $j$ for each $j \neq i$. Observe that we do not require the 
traffic matrix to be uniform all-to-all as it was done in the model studied in $[5,7]$. Our first contribution is a general characterization of the pairwise Nash topologies depending on a given traffic matrix. Our second key contribution is a new and original myopic dynamics. Since we show that the Best Response problem is NP-hard, we define a myopic dynamics which avoids to compute the best response. At each round a node is activated to improve his own benefit whenever it is possible. We define a subset of possible deviations in such a way that a sink configuration of our dynamics is a pairwise Nash configuration. And finally, our third contribution is the proof of the convergence of our dynamics to a pairwise Nash configuration for any traffic pattern under some natural assumptions on the contracting functions, including the all-to-all uniform traffic pattern. The key idea is to associate a tuple of integer values to each configuration describing some relevant parameters of the current topology in relation to the given traffic. We show that these values decrease "globally" like a potential function as the dynamics evolves until a sink configuration is reached. Under the condition that the node activation process is uniform we show that the expected convergence time is polynomial. When we consider the specific case in which the traffic matrix represents a uniform all-to-all communication pattern our dynamics converges faster than the dynamics studied by Arcaute et al. in $[5,7]$.

The paper is organized as follows. In Section 2 we define the Network Formation Game. In Section 3 we define the concept of pairwise Nash equilibrium, as well as the concept of pairwise Nash outcome and the strong notion of stable topologies for a given traffic matrix. We show a characterization of pairwise Nash topologies. In Section 4 we focus on the study of a myopic dynamics and the conditions that guarantee its convergence to pairwise Nash configurations in a polynomial number of rounds, in expectation. Finally, in Section 5 we present our conclusions and open questions.

\section{The Network Formation Game: A traffic rout- ing model}

Communication Network. We use the notation $G=(V, E)$ to denote the network topology consisting of a set $V$ of $n$ nodes and a set $E$ of edges. The $n$ nodes are the players of our game. We assume that edges or links are undirected and we denote by $i j$ the edge between $i$ and $j$. We use the shorthand $i j \in G$ to denote that $G$ contains $i j$ and use $G+i j$ and $G-i j$ to represent the resulting graph from adding $i j$ to $G$ and, respectively, subtracting $i j$ from $G$. We denote by $C_{u}$ the connected component of $G$ that contains $u \in V\left(C_{u}^{\prime}\right.$ if the graph is denoted by $G^{\prime}$ ).

Players. In our game each player $i \in V$ wants to send a certain amount of traffic to other players. Let $t_{i j} \geq 0$ be the number of units or packets that player $i$ wants to send to player $j$. Let $T=\left(t_{i j}\right)_{i, j \in V}$ be the traffic matrix and let $G_{T}=\left(V,\left\{i j: t_{i j}+t_{j i}>0\right\}\right)$ be the graph associated to $T$. We want to 
remark that $G_{T}$ does not have to be the resulting topology of the game. Let us define Participants $(i)=\left\{j: t_{i j}+t_{j i}>0\right\}$.

The strategy of each player $i$ is defined by two sets $F_{i}, T_{i} \subseteq V$ where $F_{i}$ is the set of players that $i$ is willing to accept a contract from and $T_{i}$ is the set of players that $i$ wants to contract with. Let $\Gamma=\Gamma(\mathbf{T}, \mathbf{F})$ be a directed graph representing the contracting graph which captures the direction of the contracts. A contract $(i, j) \in \Gamma$ if and only if $i \in F_{j}$ ( $j$ accepts a contract from $i$ ) and $j \in T_{i}$ ( $i$ wants to contract with $j$ ). Let $G=G(\mathbf{T}, \mathbf{F})$ be the resulting communication network defined as follows: $i j \in G$ if and only if $(i, j) \in \Gamma$ or $(j, i) \in \Gamma$.

Every player $i$ experiences three types of costs in the communication network $G$ : the routing cost $R(i ; G)$, the maintenance cost $M(i ; G)$, and the disconnection cost $D(i ; G)$. The total cost of node $i$ in a graph $G$, is defined as $C(i ; G)=R(i ; G)+M(i ; G)+D(i ; G)$.

The routing cost is defined as $R(i ; G)=c_{i} f(i ; G)$ where $c_{i}$ is the routing cost of player $i$ per unit of traffic and $f(i ; G)$ is the total traffic that transits through $i$ including the traffic received by $i$ and the traffic sent by $i$. We assume that traffic is routed uniformly across shortest paths. If there are multiple shortest paths, then the traffic is split equally among all available paths. Moreover, we consider routing policies satisfying the following flow monotonicity property: for all $G$ and for all $i$ and $j, f(i ; G) \leq f(i ; G+i j)$.

The maintenance cost is defined by $M(i ; G)=\Pi \delta(i ; G)$ where $\Pi>0$ is the cost incurred by the endpoints of each link and $\delta(i ; G)$ is the degree of node $i$ in graph $G$.

Finally, the disconnection cost $D(i ; G)$ depends on whether $i$ is connected to its participants. We do not consider any particular disconnection function but we will consider only those that satisfy some properties to be defined later. In particular we assume that the disconnection cost $D(i ; G)$ does not depend on the selected node to connect $i$ to its participants.

Assumption 1 (About disconnection): $D(i ; G)=0$ if $i$ is connected to all its participants. Otherwise, if $j \in$ Participants $(i)$ and $C_{i} \neq C_{j}, D(i ; G+i k)=$ $D(i ; G+i l)$ for any $k, l \in C_{j}$.

Contracting function and payments. A contracting function $Q$ can be interpreted as the outcome of a negotiation process that depends on the network topology. We assume that $Q(i, j ; G)$ specifies the transfer of benefit from player $i$ to player $j$ when the network topology is $G$. (If $Q(i, j ; G)<0$, then the transfer is from $j$ to $i$ ). Instead of considering particular contracting functions, we are interested in general contracting functions that satisfy some natural assumptions.

Given $Q$ and $\Gamma$, the payment matrix $\mathbf{P}=\left(p_{i j}\right)_{i, j \in V}$ specifies the payments between players. If $i=j$ we assume that $p_{i j}=0$. For each pair $i, j$ of different players, the payment from $i$ to $j$ is defined as follows:

$$
p_{i j}= \begin{cases}Q(i, j ; G) & \text { if }(i, j) \in \Gamma, \\ 0 & \text { otherwise }\end{cases}
$$


The total utility of player $i$ in the communication network $G$ with payment matrix $\mathbf{P}$ is defined by

$$
U(i ; G, \mathbf{P})=\sum_{j \neq i}\left(p_{j i}-p_{i j}\right)-C(i ; G) .
$$

We assume that the contracting function $Q$ jointly with the disconnection function $D$ define a utility function $U$ such that, for any $i$, player $i$ prefers always to include more elements of Participants $(i)$ in his connected component when possible.

As an extension of the usual notation, let $C_{i}^{1}$ and $C_{i}^{2}$ be the connected components containing $i$ in $G_{1}$ and $G_{2}$.

Assumption 2 (Monotonicity): For any player $i$ and for any pair of configurations $\left(G_{1}, \Gamma_{1}, \mathbf{P}_{1}\right)$ and $\left(G_{2}, \Gamma_{2}, \mathbf{P}_{2}\right)$, if Participants $(i) \cap C_{i} \subsetneq$ Participants $(i) \cap$ $C_{i}^{\prime}$ then $U\left(i ; G_{1}, \mathbf{P}_{1}\right)<U\left(i ; G_{2}, \mathbf{P}_{2}\right)$.

In the following section we give a natural example of $Q$ and $D$ that satisfy both assumptions. On the one hand we assume that there exists a maximum value $p_{M A X}$ bounding the payment of any link of the communication graph. On the other hand we define a disconnection function $D(i ; G)$ depending on $p_{M A X}$ and the number of nodes in Participants $(i)-C_{i}$. To our knowledge, both seem very natural conditions.

We refer to $(G(\mathbf{T}, \mathbf{F}), \Gamma(\mathbf{T}, \mathbf{F}), \mathbf{P}(\mathbf{T}, \mathbf{F}))$ as the outcome or configuration associated to strategy vectors $(\mathbf{T}, \mathbf{F})$. By an abuse of notation we will often use the shorthand $G, \Gamma$ and $\mathbf{P}$ to refer to specific instantiations of network topology $G(\mathbf{T}, \mathbf{F})$, contracting graph $\Gamma(\mathbf{T}, \mathbf{F})$, and payment matrix $\mathbf{P}(\mathbf{T}, \mathbf{F})$, respectively. We say that $(G, \Gamma, \mathbf{P})$ is a feasible configuration if there exist strategy vectors $(\mathbf{T}, \mathbf{F})$ such that $(G, \Gamma, \mathbf{P})$ is the outcome associated to $(\mathbf{T}, \mathbf{F})$. Given $(\mathbf{T}, \mathbf{F})$, the utility of node $i$ is defined by $U(i ; G(\mathbf{T}, \mathbf{F}), \mathbf{P}(\mathbf{T}, \mathbf{F}))$.

\section{A family of contracting functions and discon- necting cost functions fulfilling the utility re- quirements}

We provide a natural example of a family of contracting functions functions $Q$ and adequate disconnection costs $D$ so that the corresponding utility functions satisfy both Assumptions 1 and 2 .

Recall that a game is defined by $\left(V, T,\left(c_{i}\right)_{i \in V}, \Pi, D, Q\right)$ where $V$ is a set of $n$ players or nodes, $T$ is the traffic matrix, $c_{i}$ is the routing cost of player $i$ per unit of traffic, $\Pi$ is the maintenance cost, $D$ is the disconnection cost, and $Q$ is the contracting function. Let $C_{M A X}=\max _{i \in V}\left\{c_{i}\right\}$ and $T_{M A X}=\sum_{i, j \in V} t_{i j}$.

Recall that he total cost of player $i$ in a graph $G$ is defined by

$$
C(i ; G)=c_{i} f(i ; G)+\Pi \delta(i ; G)+D(i ; G)
$$


and the total utility of player $i$ in the communication network $G$ with payment matrix $\mathbf{P}$ is defined by

$$
U(i ; G, \mathbf{P})=\sum_{j \neq i}\left(p_{j i}-p_{i j}\right)-C(i ; G)
$$

Our bounded payment contracting function family requires that $Q$ satisfies the property that the payment of any link can no be greater than a certain maximum value. Formally, we consider contracting functions $Q$ for which there exists an upper limit $P_{M A X}>0$ such that $\forall G, \forall i, j \in V(G),|Q(i, j ; G)| \leq$ $P_{M A X}$.

The disconnection cost $D$, for a given contracting function with payment bound $P_{M A X}$, is defined as

$$
D(i ; G)=\| \text { Participants }(i)-C_{i} \| V_{M A X}
$$

where $V_{M A X}=C_{M A X} T_{M A X}+n \Pi+2 n P_{M A X}$.

It is easy to see that $D$ satisfies Assumption 1 as the disconnection cost of a node $i$ directly depends on the number of participants of $i$ that are not connected to $i$. Formally, we have that if Participants $(i) \subseteq C_{i}$ then $D(i ; G)=0$. Moreover, if $j \notin C_{i}$ and $j \in \operatorname{Participants}(i)$ then if we consider any pair $k, l \in C_{j}$ , the graphs $G+i k$ and $G+i l$ have the same new connected component containing $i$. Hence $D(i ; G+i k)=D(i ; G+i l)$.

It only remains to show that the corresponding utility function $U$ satisfies Assumption 2. Let us consider a pair of configurations $(G, \Gamma, P)$ and $\left(G^{\prime}, \Gamma^{\prime}, P^{\prime}\right)$ such that $C_{i} \cap$ Participants $(i) \subsetneq C_{i}^{\prime} \cap$ Participants $(i)$.

Let $K=\|$ Participants $(i)-C_{i} \|$ and $K^{\prime}=\|$ Participants $(i)-C_{i}^{\prime} \|$. Notice that $K>K^{\prime}$ and $D(i, G)=K V_{M A X}$ and $D\left(i ; G^{\prime}\right)=K^{\prime} V_{M A X}$.

Now let us consider the value of the utility function, for node $i$, in both graphs. Observe that we have $U(i ; G, P) \leq P_{M A X}(n-1)-C(i ; G)$ and that $U\left(i ; G^{\prime}, P^{\prime}\right) \geq-C\left(i ; G^{\prime}\right)$. If we consider their difference, we have

$$
\begin{aligned}
U(i ; G, P) & -U\left(i ; G^{\prime}, P^{\prime}\right) \leq 2 P_{M A X}(n-1)-C(i ; G)+C\left(i ; G^{\prime}\right) \\
& \leq 2 P_{M A X}(n-1)+\Pi \delta\left(i ; G^{\prime}\right)+c_{i} f\left(i ; G^{\prime}\right)-D(i ; G)+D\left(i ; G^{\prime}\right) \\
& \leq 2 P_{M A X}(n-1)+\Pi \delta\left(i ; G^{\prime}\right)+c_{i} f\left(i ; G^{\prime}\right)-\left(K-K^{\prime}\right) V_{M A X} \\
& \leq 2 P_{M A X}(n-1)+\Pi(n-1)+C_{M A X} T_{M A X}-V_{M A X} \\
& <2 P_{M A X} n+\Pi n+C_{M A X} T_{M A X}-V_{M A X} \\
& =0 .
\end{aligned}
$$

Hence, for each pair of configurations $(G, \Gamma, P)$ and $\left(G^{\prime}, \Gamma^{\prime}, P^{\prime}\right)$ for which $C_{i} \cap$ Participants $(i) \subset C_{i}^{\prime} \cap$ Participants $(i)$, we have that

$$
U(i ; G, P)-U\left(i ; G^{\prime}, P^{\prime}\right)<0 \text {. }
$$

\section{Pairwise Nash Equilibrium}

One of the aims of our model is to formalize the fact that players may deviate from their strategies not only unilaterally, but also bilaterally. It seems natural 
that a player can delete contracts unilaterally. Once a contract is deleted, the corresponding network link disappears. But if a player wants to add a new contract with another player, then the former needs the agreement of the latter. This bilateral process leads to the creation of a new link between both players. Since Nash equilibrium does not allow us to represent the link formation as a bilateral process, we adopt the notion of pairwise Nash stability used in [6].

Given the strategy vector $(\mathbf{T}, \mathbf{F})$, let $G=G(\mathbf{T}, \mathbf{F}), \Gamma=\Gamma(\mathbf{T}, \mathbf{F})$, and $\mathbf{P}=$ $\mathbf{P}(\mathbf{T}, \mathbf{F})$. Given the strategy vector $\left(\mathbf{T}^{\prime}, \mathbf{F}^{\prime}\right)$, let $G^{\prime}=G\left(\mathbf{T}^{\prime}, \mathbf{F}^{\prime}\right)$ and $\Gamma^{\prime}=$ $\Gamma\left(\mathbf{T}^{\prime}, \mathbf{F}^{\prime}\right)$. We define $\mathbf{P}^{\prime}=\left(p_{i j}^{\prime}\right)_{i, j \in V}$ according to:

$$
p_{k l}^{\prime}= \begin{cases}p_{k l} & \text { if }(k, l) \in \Gamma^{\prime} \text { and }(k, l) \in \Gamma \\ Q\left(k, l ; G^{\prime}\right) & \text { if }(k, l) \in \Gamma^{\prime} \text { and }(k, l) \notin \Gamma \\ 0 & \text { otherwise }\end{cases}
$$

We assume that all contracts present both before and after the deviation maintain the same payment which is consistent with the notion of contract.

Pairwise Nash Equilibrium. Let us consider a game defined by a set of players $V$, a traffic matrix $T$, and a utility function $U$ with parameters $c_{i}$ for each player $i, \Pi, Q$, and $D$ (satisfying Assumptions 1 and 2). A strategy vector $(\mathbf{T}, \mathbf{F})$ is a pairwise Nash equilibrium for the game defined by $\left(V, T,\left(c_{i}\right)_{i \in V}, \Pi, D, Q\right)$ if

(1) No unilateral deviation is profitable, i.e., for all $i$ and for all $\left(T_{i}^{\prime}, F_{i}^{\prime}\right) \subseteq\left(T_{i}, F_{i}\right), U(i ; G, \mathbf{P}) \geq U\left(i ; G^{\prime}, \mathbf{P}^{\prime}\right)$.

(2) No bilateral deviation is profitable, i.e., for all $i, j$ and for all $\left(T_{i}, F_{i}\right) \subseteq\left(T_{i}^{\prime}, F_{i}^{\prime}\right)$ and $\left(T_{j}, F_{j}\right) \subseteq\left(T_{j}^{\prime}, F_{j}^{\prime}\right)$, if $U(i ; G, \mathbf{P})<U\left(i ; G^{\prime}, \mathbf{P}^{\prime}\right)$ then $U(j ; G, \mathbf{P})>U\left(j ; G^{\prime}, \mathbf{P}^{\prime}\right)$.

In a pairwise Nash equilibrium $(\mathbf{T}, \mathbf{F})$, the corresponding communication network $G=G(\mathbf{T}, \mathbf{F})$ does not change. In this situation no player has incentive to deviate. In section 4 we define a dynamic model where different networks are formed over time. For this reason we consider that at each round, the activated player has to update the payments of all his links according to the present topology.

Pairwise Nash Outcome. A feasible configuration $(G, \Gamma, \mathbf{P})$ is a pairwise Nash outcome for the game defined by $\left(V, T,\left(c_{i}\right)_{i \in V}, \Pi, D, Q\right)$ if there exists a pairwise Nash equilibrium $(\mathbf{T}, \mathbf{F})$ for such a game such that $(G, \Gamma, \mathbf{P})$ is the outcome of $(\mathbf{T}, \mathbf{F})$.

Notice that a configuration $(G, \Gamma, \mathbf{P})$ can be the outcome of different strategy vectors, even the contracting graph $\Gamma$ can be the result of different strategy vectors. Let us define a notion of normalized vector in order to associate to each $(G, \Gamma, \mathbf{P})$ a unique strategy vector. A strategy vector (NT, NF) is the normalized strategy vector of $(G, \Gamma, \mathbf{P})$ when for each player $i, N T_{i}=\{j \mid(i, j) \in$ $\Gamma\}$ and $N F_{i}=\{j \mid(j, i) \in \Gamma\}$. We can extend the definition of normalized strategy vector of a configuration $(G, \Gamma, \mathbf{P})$ to the normalized strategy vector of a contracting graph $\Gamma$ or even of a strategy vector $(\mathbf{T}, \mathbf{F})$. 
By definition, if only one of the strategy vectors producing $(G, \Gamma, \mathbf{P})$ is a pairwise Nash equilibrium then $(G, \Gamma, \mathbf{P})$ is a pairwise Nash outcome. In the following we show that the pairwise Nash stability of an outcome is equivalent to the pairwise Nash stability of its normalized strategy vector.

Lemma 1. If $(\mathbf{T}, \mathbf{F})$ is a pairwise Nash equilibrium then its corresponding normalized strategy vector (NT, NF) also is a pairwise Nash equilibrium. However, there exists a non pairwise Nash equilibrium $(\mathbf{T}, \mathbf{F})$ such that its corresponding normalized strategy vector (NT, NF) is a pairwise Nash equilibrium.

Proof. For the first statement, let $(\mathbf{T}, \mathbf{F})$ be a strategy vector and let (NT, NF) be its corresponding normalized vector. Let $(G, \Gamma, \mathbf{P})$ be the outcome of $(\mathbf{T}, \mathbf{F})$, by the definition of normalized strategy vector, we have that $(\mathbf{T}, \mathbf{F})$ has the same outcome $(G, \Gamma, \mathbf{P})$. In the following we show that any profitable deviation of a player in (NT, NF) can be translated to a profitable deviation in $(\mathbf{T}, \mathbf{F})$.

Let us suppose that (NT, NF) is not a pairwise Nash equilibrium. Then there exists a player $i$ that prefers to deviate his strategy unilaterally or bilaterally. In the former, there exist $N T_{i}^{\prime} \subseteq N T_{i}$ and $N F_{i}^{\prime} \subseteq N F_{i}$ such that $U\left(i ; G^{\prime}, \mathbf{P}^{\prime}\right)>U(i ; G, \mathbf{P})$. The same player $i$ in $(\mathbf{T}, \mathbf{F})$ can consider a deviataion $T_{i}^{\prime}=N T_{i}^{\prime}$ and $F_{i}^{\prime}=N F_{i}$ (recall that $N T_{i}^{\prime} \subseteq N T_{i} \subseteq T_{i}$ and $N F_{i}^{\prime} \subseteq N F_{i} \subseteq F_{i}$ ). Notice that the outcome of $\left(\mathbf{T}^{\prime}, \mathbf{F}^{\prime}\right)$ is equal to the outcome of $\left(\mathbf{N T}^{\prime}, \mathbf{N F}^{\prime}\right)$ and then this unilateral deviation is also profitable for player $i$ in $(\mathbf{T}, \mathbf{F})$.

In the case of a profitable bilateral deviation of player $i$ in $(\mathbf{N T}, \mathbf{N F})$ there exists a player $j$ such that accepts a contract from $i$ (or to $i$ ). Let us suppose that $N T_{i} \cup\{j\} \subseteq N T_{i}^{\prime}, N F_{j} \cup\{i\} \subseteq N F_{j}^{\prime}, U\left(i ; G^{\prime}, \mathbf{P}^{\prime}\right)>U(i ; G, \mathbf{P})$ and $U\left(j ; G^{\prime}, \mathbf{P}^{\prime}\right) \geq$ $U(j ; G, \mathbf{P})$. Notice that $\Gamma^{\prime}=\Gamma \cup\{(i, j)\}$. Now, if we consider strategy vector $(\mathbf{T}, \mathbf{F})$, players $i$ and $j$ also prefer to deviate their strategies so that $T_{i}^{\prime}=T_{i} \cup\{j\}$ and $F_{j}^{\prime}=F_{j} \cup\{i\}$. Since the outcome of $\left(\mathbf{T}^{\prime}, \mathbf{F}^{\prime}\right)$ is equal to the outcome of $\left(\mathbf{N T}^{\prime}, \mathbf{N F}^{\prime}\right)$ then such bilateral deviation is profitable.

To prove the second statement, let us consider a contracting graph $\Gamma$ with $E(\Gamma)=\{(i, l),(i, k),(k, j)\}$ and let $(\mathbf{N T}, \mathbf{N F})$ be its normalized strategy vector. We define $(\mathbf{T}, \mathbf{F})$ as the strategy with the same contracting graph $\Gamma$ but it differs from (NT, NF) in that $j \in F_{l}$ and $j \notin N F_{l}$. Notice that if $T_{j}^{\prime}=T_{j} \cup\{l\}$ a new contract $(l, j)$ is added in the case that $j$ increases its profit and $i$ has at least the same profit. If player $j$ in $(\mathbf{N T}, \mathbf{N F})$ includes $l$ in $N T_{j}^{\prime}$ then the contracting graph does not change and the deviation is not profitable.

Proposition 1. A configuration $(G, \Gamma, \mathbf{P})$ is a pairwise Nash outcome for the game defined by $\left(V, T,\left(c_{i}\right)_{i \in V}, \Pi, D, Q\right)$ if and only if the corresponding normalized vector (NT, NF) is a pairwise Nash equilibrium.

Proof. By definition, if $(G, \Gamma, \mathbf{P})$ is the outcome of a pairwise Nash equilibrium $(\mathbf{N T}, \mathbf{N F})$ then $(G, \Gamma, \mathbf{P})$ is a pairwise Nash outcome. And by Lemma 1 if $(G, \Gamma, \mathbf{P})$ is a pairwise Nash outcome then its normalized strategy vector is a pairwise Nash equilibrium.

Notice that a normalized vector $(\mathbf{N T}, \mathbf{N F})$ is a pairwise Nash equilibrium if and only if 
1. no player can increase his utility by breaking some of his contracts, and

2. no pair of players $i, j$ can simultaneously increase their utility by creating a new link $i j$.

We are interested in characterizing the topologies that, independently of the other parameters, lead to a pairwise Nash equilibrium configuration. We say that $(G, \Gamma, \mathbf{P})$ is a proper configuration when for each $i j \in G,(i, j) \in \Gamma$ if and only if $(j, i) \notin \Gamma$. Notice that if a configuration is not proper, an existing contract may be broken without changing the topology.

Pairwise Nash Topology. A graph $G$ is a pairwise Nash topology for a traffic matrix $T$ if for all $\left(\left(c_{i}\right)_{i \in V}, \Pi, D, Q\right)$ (satisfying Assumptions 1 and 2), any feasible and proper configuration $(G, \Gamma, \mathbf{P})$ is a pairwise Nash outcome for the game defined by $\left(V, T,\left(c_{i}\right)_{i \in V}, \Pi, D, Q\right)$.

Theorem 1. A network topology $G$ is a pairwise Nash topology for a traffic matrix $T$ if and only if $G$ satisfies the following properties:

1. For every pair $u, v \in G$ such that $u \in \operatorname{Participants}(v)$ then $C_{u}=C_{v}$.

2. $G$ is a forest.

3. For every edge $u v \in G$, if $G^{\prime}=G-u v$, and $C_{u}^{\prime}, C_{v}^{\prime}$ are the corresponding connected components containing $u$ and $v$, respectively, then, $C_{u}^{\prime} \cap \operatorname{Participants}(v) \neq \emptyset$ and $C_{v}^{\prime} \cap \operatorname{Participants}(u) \neq \emptyset$.

Proof. Let $G$ be a pairwise Nash topology for $T$ :

(1) Let us suppose that there exists a pair $u, v \in V$ such that $v \in \operatorname{Participants}(u)$ and $C_{u} \neq C_{v}$. Then by Assumption 2, $u$ and $v$ prefer to create the link $u v$ to increment their utilities.

(2) If there exists a link $u v \in G$ contained in a cycle then for some contracting function we have that $p_{u v}-p_{v u}>0$. Note that if $u$ eliminates $u v$ (breaking the contract $(u, v)$ or $(v, u))$ then $u$ still remains connected to its participants. Then the maintenance cost decreases by $\Pi$, the total traffic through $u$ in $G-u v$ is not greater than that the one through $u$ in $G$ and the disconnection cost is the same by Assumption 1. Therefore node $u$ can increment its utility applying such unilateral deviation.

(3) Let us suppose that there exist $u \in V$ and $u v \in G$ such that $u$ remains connected to all its participants in $G-u v$. Hence, for a contracting function with $p_{u v}-p_{v u}>0, u$ can increase its utility by eliminating the link $u v$. Let $G$ be a topology that satisfies (1), (2) and (3). Let us consider a proper and feasible configuration $(G, \Gamma, \mathbf{P})$. If $u$ breaks a contract then by (3) $u$ becomes disconnected from some of its participants and then, by Assumption 2 this unilateral deviation is not profitable. Moreover if a new link $u v$ is created, then if we consider a contracting function such that $p_{u v}^{\prime}-p_{v u}^{\prime}>0$ (exactly one contract either $(u, v)$ or $(v, u)$ is added) we have that the utility of $u$ is decreased strictly since and $p_{u v}=p_{v u}=0$, the maintenance cost increases $(\Pi>0)$ and the routing cost of $u$ may also be incremented. Notice that by (1) and (2) a new link $u v$ can provide shorter paths between pairs of participants. 

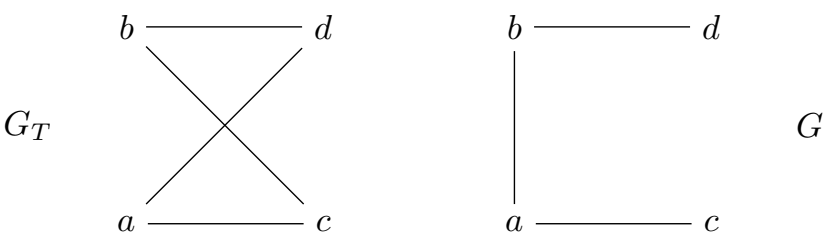

Figure 1: $G$ is a pairwise Nash topology of a traffic matrix $T$

Given the above characterization it is not hard to see that, for any traffic matrix $T$, every spanning forest of $G_{T}$ is a pairwise Nash topology, but there might exist pairwise Nash topologies $G \neq G_{T}$. One example is the graph $G$ given in Fig. 1. When $G_{T}$ is a complete graph, then we obtain as a corollary an analogous result to the one presented in [5] for the uniform all-to-all traffic matrix, i.e., for any $i \neq j, t_{i, j}=1$.

Corollary 1. Let $T$ be a traffic matrix such that $G_{T}$ is a complete graph. $G$ is a pairwise Nash topology for $T$ if and only if $G$ is a tree containing all the nodes of $G_{T}$.

\section{$5 \quad$ Myopic Dynamics}

We consider a dynamic model of the previous network formation game in which different networks are formed over time. The dynamic game can be seen as a process where players interact over time through unilateral or bilateral deviations. At each round a player can change his strategy only if this change improves his current payoff. Our aim is to define a myopic dynamics that converges to a pairwise Nash equilibria in polynomial time. Note that a strategic deviation consists in either deleting unilaterally a set of contracts or adding bilaterally a new link. Hence the set of all possible deviations has an exponential size with respect to the number of players. We show that the problem of computing the best possible deviation is NP-hard.

BEST RESPONSE: Given a game defined by $\left(V, T,\left(c_{i}\right)_{i \in V}, \Pi, D, Q\right)$, a configuration $(G, \Gamma, \mathbf{P})$, a node $u$, and an integer $C$, decide whether there is a deviation of $u$ such that after applying it, $u$ 's utility is greater than or equal to $C$.

Theorem 2. The BEST RESPONSE problem is NP-complete.

Proof. It is not hard to see that BR is in NP. We can define a nondeterministic algorithm that guesses a deviation of player $u$ and afterwards it verifies that the utility of player $u$ is greater than or equal to $C$. Of course, we assume that given the description of $D$ and $Q$, both functions are polynomial time computable in $n$, the number of players.

In order to prove the NP hardness we reduce the Independent Set problem to BR. Given a graph $G=(V, E)$ and an integer $C$ such that $0 \leq C \leq|V|$, the reduction function returns a configuration $(S, \Gamma, \mathbf{P})$, a game $\left(V, T,\left(c_{i}\right)_{i \in V}, \Pi, D, Q\right)$, a new node $u$, and the value $C$ where: 
- $S$ is a star where $V(S)=V \cup\{u\}$, being $u \notin V$ and $E(S)=\{u v: v \in V\}$.

- $T=\left(t_{v w}\right)$ where $t_{v w}=1$ if $v w \in E$, and $t_{v w}=0$, otherwise. Since $G$ is undirected, if $v w \in E$, we assume that only one of $t_{v w}$ and $t_{w v}$ has the value 1 and $t_{v w}+t_{w v}=1$. Note that Participants $(u)=\emptyset$.

$-\Gamma=\{(v, u): v \in V\}$.

- $Q(v, u ; G)=2$ and $Q(u, v ; G)=-2$ for all $v \in V$.

- $D$ is any function such that jointly with $Q$ satisfy Assumptions 1 and 2 (Since $u$ has no participants, $D(u ; G, \mathbf{P})=0$ whatever be $G$ and $\mathbf{P})$.

- The maintenance cost $\Pi=1$ and the routing $\operatorname{costs} c_{v}=n$ for all $v \in$ $V \cup\{u\}$.

Notice that since $u$ has a contract to each of the $n$ nodes of $V$, then the only possible deviations of $u$ consist in breaking contracts. Let us assume that there is an independent set $I \subseteq V$ such that $|I|=C$. If $u$ deletes all the contracts with $I^{\prime}=\{v: v \notin I\}$ then $u$ only remains connected to all nodes of $I$. Let $S^{\prime}$ be the resulting topology and $\mathbf{P}^{\prime}$ the resulting payment matrix. Since there is no traffic between the nodes in $I$, then $U\left(u, S^{\prime}, \mathbf{P}^{\prime}\right)=2|I|-|I|=|I|=C$.

Conversely, let us assume that there is no independent set of $G$ of size $C$. Then, for all $I \subseteq V$ with $|I| \geq C$, we have that there exists at least a pair of nodes $v, w \in I$ such that $v w \in E$. If after the deviation of $u$ there still remain $a<C$ links between $u$ and nodes in $V$, the resulting graph $S^{\prime}$ and payment matrix $\mathbf{P}^{\prime}$ satisfy $U\left(u, S^{\prime}, \mathbf{P}^{\prime}\right)=2 a-a-c_{u} k$ where $k=\sum_{v, w \in I, v w \in E} t_{v w} \geq 0$. Hence, $U\left(u, S^{\prime}, \mathbf{P}^{\prime}\right) \leq a<C$. Otherwise, if after the deviation of player $u$ there are $b \geq C$ links between $u$ and nodes in $V$, then the resulting graph $S^{\prime}$ and payment matrix $\mathbf{P}^{\prime}$ satisfy $U\left(u, S^{\prime}, \mathbf{P}^{\prime}\right)=2 b-b-c_{u} k$ where $k=\sum_{v, w \in I, v w \in E} t_{v w} \geq 1$. Since $c_{u}=n$ then $U\left(u, S^{\prime}, \mathbf{P}^{\prime}\right)<0$.

Therefore, the NP-hardness of the BEST RESPONSE problem leads us to reconsider the definition of each round of the dynamics. The main idea behind our definition is to restrict the best response so that the deviation of the active node can be computed in polynomial time and when no deviation of this kind is possible, then the current configuration is a pairwise Nash configuration.

Myopic dynamics We consider a discrete dynamics where the time is divided into periods or rounds. We denote by $\left(G^{(k)}, \Gamma^{(k)}, \mathbf{P}^{(k)}\right)$ the configuration at the beginning of round $k$, for any $k \geq 0$. We assume that any initial configuration $\left(G^{(0)}, \Gamma^{(0)}, \mathbf{P}^{(0)}\right)$ is a feasible and proper configuration.

At each round $k$ a node $u$ is activated by an activation process. An activation process is any discrete time stochastic process $\left\{U_{k}\right\}_{k \in \mathbb{N}}$ where all $U_{k}$ are i.i.d. random variables from $V$ drawn with full support. A realization of an activation process is called an activation sequence. We consider a uniform activation process, where the probability that $U_{k}=u$ is uniform over all nodes. Thus $P\left[U_{k}=u\right]=1 / n$.

In a single round $k$, if the active node is $u_{k}$ and the current configuration is $\left(G^{(k)}, \Gamma^{(k)}, \mathbf{P}^{(k)}\right)$ then $u_{k}$ takes the following actions: 
1. $u_{k}$ updates the prices of all its current contracts: $p_{u_{k} v}^{(k)}=Q\left(u_{k}, v ; G^{(k)}\right)$ if $\left(u_{k}, v\right) \in \Gamma^{(k)}$ and $p_{v u_{k}}^{(k)}=Q\left(v, u_{k} ; G^{(k)}\right)$ if $\left(v, u_{k}\right) \in \Gamma^{(k)}$.

2. $u_{k}$ selects the action from all the possible actions described below that maximizes its utility. If $u_{k}$ has multiple choices, then we assume that $u_{k}$ selects one of them randomly. The possible actions are:

(i) To break one of its contracts $\left(u_{k}, v\right)$ (or $\left.\left(v, u_{k}\right)\right)$. Therefore $\Gamma^{(k+1)}=$ $\Gamma^{(k)}-\left(u_{k}, v\right)\left(\right.$ or $\left.\Gamma^{(k+1)}=\Gamma^{(k)}-\left(v, u_{k}\right)\right), G^{(k+1)}=G^{(k)}-u_{k} v$ and $\mathbf{P}^{(k+1)}=\mathbf{P}^{(k)}$.

(ii) To ask for a new contract $\left(u_{k}, v\right)$, and if $v$ accepts, then $\Gamma^{(k+1)}=$ $\Gamma^{(k)}+\left(u_{k}, v\right), G^{(k+1)}=G^{k}+u_{k} v$ and $\mathbf{P}^{(k+1)}=\mathbf{P}^{(k)}$.

(iii) To do nothing, and then $\left(G^{(k+1)}, \Gamma^{(k+1)}, \mathbf{P}^{(k+1)}\right)=\left(G^{(k)}, \Gamma^{(k)}, \mathbf{P}^{(k)}\right)$.

Let MyopicRound be the function defined as follows: Given as input a node $u$ and a configuration $(G, \Gamma, \mathbf{P})$, MyopicRound $(u, G, \Gamma, \mathbf{P})$ returns the configuration resulting of applying 1 ) and 2) considering that the active node is $u$ and the current configuration is $(G, \Gamma, \mathbf{P})$. Hence given an initial configuration $\left(G^{(0)}, \Gamma^{(0)}, \mathbf{P}^{(0)}\right)$ and an activation sequence $u_{0} \ldots, u_{k} u_{k+1} \ldots$, we have that for each $k \geq 0,\left(G^{(k+1)}, \Gamma^{(k+1)}, \mathbf{P}^{(k+1)}\right)=$ MyopicRound $\left(u_{k}, G^{(k)}, \Gamma^{(k)}, \mathbf{P}^{(k)}\right)$.

It is not hard to see that for any $k \geq 0,\left(G^{(k)}, \Gamma^{(k)}, \mathbf{P}^{(k)}\right)$ is a proper configuration. Given any initial configuration $\left(G^{(0)}, \Gamma^{(0)}, \mathbf{P}^{(0)}\right)$ and an instance of the activation process, we say that the dynamics converges if there exists $K$ such that for all $k>K,\left(G^{(k+1)}, \Gamma^{(k+1)}, \mathbf{P}^{(k+1)}\right)=\left(G^{(k)}, \Gamma^{(k)}, \mathbf{P}^{(k)}\right)$. Further, we say that the dynamics converges uniformly if for every $\epsilon>0$ there exists $K$ such that

$$
\operatorname{Pr}\left[\left(G^{(k+1)}, \Gamma^{(k+1)}, \mathbf{P}^{(k+1)}\right)=\left(G^{(k)}, \Gamma^{(k)}, \mathbf{P}^{(k)}\right), \forall k>K\right] \geq 1-\epsilon
$$

where the probability is taken with respect to the activation process.

A configuration $(G, \Gamma, \mathbf{P})$ is a sink configuration of the dynamics if whatever is the node $u$, MyopicRound $(u, G, \Gamma, \mathbf{P})=(G, \Gamma, \mathbf{P})$. A key property of our dynamics is that any sink configuration also is a pairwise Nash outcome or configuration. Since we assume that $\Pi>0$, it is not hard to see that the topology of a sink configuration has no cycles. The hard part of the proof is to show that in any sink configuration any node does not have incentive to break a set of at least two contracts.

Proposition 2. Any sink configuration is a pairwise Nash configuration.

Proof. Let $(G, \Gamma, \mathbf{P})$ be a sink configuration. Then any node is connected to its participants. Hence for any $u \in V, D(u ; G)=0$.

Let us suppose that $G$ contains a cycle and let $u v$ be a link of such cycle. Since $(G, \Gamma, \mathbf{P})$ is a proper configuration either $(u, v) \in \Gamma$ or $(v, u) \in \Gamma$. Let us assume w.l.o.g. that $(u, v) \in \Gamma$ and then $p_{v u}=0$. If $p_{u v}>0$ then

$$
U(u ; G, \mathbf{P})-U\left(u ; G-u v, \mathbf{P}^{\prime}\right)=-p_{u v}-\Pi-c_{u}(f(u, G)-f(u ; G-u v)) .
$$


Since $f(u, G)-f(u ; G-u v) \geq 0$ we have that $U(u ; G, \mathbf{P})-U\left(u ; G-u v, \mathbf{P}^{\prime}\right)<0$. Then $u$ has incentive to break the link $u v$, which contradicts the assumption that $(G, \Gamma, \mathbf{P})$ is a sink configuration. In the case that $p_{u v}<0$ we obtain that $v$ has incentive to break $u v$ (using the same arguments but interchanging $u$ with $v)$.

From now on we have that $G$ has no cycle and $\forall u, D(u ; G)=0, u$ does not want to break any link $u v$, and if $u$ has incentive to add a new link $u w$, then $w$ does not agree. It remains to show that no node $u$ has incentive to break a set of links (of cardinality greater than 1).

Let us suppose that $\exists u, \exists A=\left\{u v_{1}, v v_{2}, \ldots, u v_{k}\right\} \subseteq E$ a minimal set with $k \geq 2$ satisfying $U(u ; G, \mathbf{P})<U\left(u ; G-A, \mathbf{P}^{\prime}\right)$. Therefore,

$$
\sum_{v \mid u v \in A}\left(p_{v u}-p_{u v}\right)<\Pi|A|+c_{u}(f(u ; G)-f(u ; G-A))
$$

Intuitively, $u$ has an incentive to break all the links of $A$ since the payments that $u$ receives do not amortize their maintenance and the routing costs.

Moreover, we know that every node has no incentive to break only one of its links, i.e. $\forall u \in V, \forall u v \in E, U(u ; G, \mathbf{P}) \geq U\left(u ; G-u v, \mathbf{P}^{\prime}\right)$. Then we have that:

$$
\begin{aligned}
\left(p_{v u}-p_{u v}\right)-\Pi \delta(u ; G)-c_{u} f(u ; G) & \geq-\Pi(\delta(u ; G)-1)-c_{u} f(u ; G-u v) \\
p_{v u}-p_{u v} & \geq \Pi+c_{u}(f(u ; G)-f(u ; G-u v)) \\
p_{v u}-p_{u v} & \geq \Pi
\end{aligned}
$$

Let us consider the links of $A$. It can be the case that $u$ does not route traffic through any link $u v \in A, f(u ; G)=f(u ; G-u v)$. Then we have that

$$
\begin{aligned}
\operatorname{by}(2), \sum_{u v \in A}\left(p_{v u}-p_{u v}\right) & \geq \Pi|A| \\
\text { and by }(1), \sum_{u v \in A}\left(p_{v u}-p_{u v}\right) & <\Pi|A|+c_{u}(f(u ; G)-f(u, G-A))=\Pi|A|
\end{aligned}
$$

which is a contradiction.

If it is the case that there exists $u v \in A$ such that $f(u ; G)>f(u ; G-u v)$ then let us consider the subset of $A$ defined by $A^{\prime}=A-\{u v\}$ and let $A^{\prime \prime}=$ $\{u w \in E \mid u w \notin A\}$ (Notice that $A^{\prime} \cup\{u v\} \cup A^{\prime \prime}=\{u w \mid w \in V\} \cap E$ ). Let $B^{\prime}=\left\{w \mid u w \in A^{\prime}\right\}$ and $B^{\prime \prime}=V-(B \cup\{u, v\})$, and let us consider the different kind of traffic through $u$. We denote by:

- $f_{B^{\prime \prime} B^{\prime \prime}}$ the amount of traffic through $u$ between pairs of nodes of $B^{\prime \prime}$,

- $f_{v B^{\prime \prime}}$ the amount of traffic through $u$ between $v$ and $B^{\prime \prime}$,

- $f_{v B^{\prime}}$ the amount of traffic through $u$ between $v$ and $B^{\prime}$, 
- $f_{B^{\prime} B^{\prime}}$ the amount of traffic through $u$ between pairs of nodes of $B^{\prime}$, and

- $f_{B^{\prime} B^{\prime \prime}}$ the amount of traffic through $u$ between $B^{\prime}$ and $B^{\prime \prime}$.

Notice that $f_{B^{\prime \prime} B^{\prime \prime}}, f_{v B^{\prime \prime}}, f_{v B^{\prime}}, f_{B^{\prime} B^{\prime}}, f_{B^{\prime} B^{\prime \prime}} \geq 0$. We can express the traffic through $u$ as follows:

$$
\begin{aligned}
f(u ; G) & =f_{v B^{\prime \prime}}+f_{v B^{\prime}}+f_{B^{\prime} B^{\prime}}+f_{B^{\prime} B^{\prime \prime}}+f_{B^{\prime \prime} B^{\prime \prime}} \\
f(u ; G-u v) & =f_{B^{\prime} B^{\prime}}+f_{B^{\prime} B^{\prime \prime}}+f_{B^{\prime \prime} B^{\prime \prime}} \\
f\left(u ; G-A^{\prime}\right) & =f_{v B^{\prime \prime}}+f_{B^{\prime \prime} B^{\prime \prime}} \\
f(u ; G-A) & =f_{B^{\prime \prime} B^{\prime \prime}}
\end{aligned}
$$

Since $A$ is a minimal set satisfying $U(u ; G, \mathbf{P})<U\left(u ; G-A, \mathbf{P}^{\prime}\right)$ then $U(u ; G, \mathbf{P}) \geq$ $U\left(u ; G-A^{\prime}, \mathbf{P}^{\prime}\right)$ and $U(u ; G, \mathbf{P}) \geq U\left(u ; G-u v, \mathbf{P}^{\prime}\right)$. Thus

$$
\begin{aligned}
\sum_{u w \in A^{\prime}}\left(p_{w u}-p_{u w}\right) & \geq \Pi(|A|-1)+c_{u}\left(f_{v B^{\prime}}+f_{B^{\prime} B^{\prime}}+f_{B^{\prime} B^{\prime \prime}}\right) \\
p_{v u}-p_{u v} & \geq \Pi+c_{u}\left(f_{v B^{\prime}}+f_{v B^{\prime \prime}}\right)
\end{aligned}
$$

Combining both inequalities we have that

$$
\begin{aligned}
\sum_{u w \in A}\left(p_{w u}-p_{u w}\right) & \geq \Pi|A|+c_{u}\left(2 f_{v B^{\prime}}+f_{v B^{\prime \prime}}+f_{B^{\prime} B^{\prime}}+f_{B^{\prime} B^{\prime \prime}}\right) \\
\text { and by (1), } \sum_{u w \in A}\left(p_{w u}-p_{u w}\right) & <\Pi|A|+c_{u}\left(f_{v B^{\prime}}+f_{v B^{\prime \prime}}+f_{B^{\prime} B^{\prime}}+f_{B^{\prime} B^{\prime \prime}}\right)
\end{aligned}
$$

Hence $2 f_{v B^{\prime}}<f_{v B^{\prime}}$ which is a contradiction since $f_{v B^{\prime}} \geq 0$. The contradiction arises from considering that there exists a minimal set $A$ with $|A| \geq 2$ satisfying $U(u ; G, \mathbf{P})<U\left(u ; G-A, \mathbf{P}^{\prime}\right)$. Then, no node $u$ has incentive to break any set of links.

In order to guarantee the convergence of our dynamics we consider a class of contracting functions satisfying two natural properties. First we assume that the contracting function is anti-symmetric in the sense that $Q(i, j ; G)$ and $Q(j, i ; G)$ have the same magnitude but opposite signs. The idea is that a link only is payed by one of the two endpoints. And second we focus on the convenience of a node of being connected to the maximum number of its participants.

Anti-symmetric property: A contracting function $Q$ is anti-symmetric if, for all nodes $i$ and $j$, and any graph $G, Q(i, j ; G)=-Q(j, i ; G)$.

Affine property: A contracting function $Q$ is affine if, for all nodes $i, j, k$ such that $j \in$ Participants(i) and $k \notin$ Participants $(i)$, then we have that $|Q(i, j ; G+i j)|>|Q(i, k ; G+i k)|$.

Now in addition to Assumptions 1 and 2 we also require that the contracting function should satisfy the anti-symmetric and affine properties. One example of contracting function with payment bounded by 2 , satisfying the two additional properties, is the following: 


$$
\text { If } i<j, Q(i, j, G)=\left\{\begin{array}{cc}
2 & \text { if } j \in \text { Participants }(i) \\
1 & \text { otherwise }
\end{array}\right.
$$

And

$$
\text { If } i>j, Q(i, j, G)= \begin{cases}-2 & \text { if } j \in \operatorname{Participants}(i) \\ -1 & \text { otherwise }\end{cases}
$$

Observe that this contracting function provides an example in which the player with the lowest identity pays for the established link.

In the rest of this section we introduce all the ingredients to prove the convergence of our dynamics when the contracting function is antisymmetric and affine. From now on let us suppose that $Q$ is an antisymmetric and affine contracting function. The main idea of the proof is based on associating a tuple of integer values to each configuration. The components of such tuple describe some important parameters of the current topology in relation to the traffic matrix. We are going to show that the values of these parameters decrease "globally" as the dynamics evolves, like a kind of potential function. In fact we prove that at each round, some parameters never increase and in the case that the current configuration is not a pairwise Nash equilibrium, then some parameters decrease strictly. Finally, since the configurations with "minimum values" are pairwise Nash stable, then we can conclude that our dynamics converges. Let us introduce formally the key element of the proof and all the properties needed to prove the dynamics convergence.

The mapping of game configurations We define the mapping function $\Phi$, a function that maps game configurations to 4-tuples of non-negative integers, as follows. $\Phi(G, \Gamma, \mathbf{P})=\left(C_{F}, C_{E}, A_{E}, A_{P}\right)$, where:

- $C_{F}$ is the number of edges that have to be removed to have a spanning forest of $G$.

- $C_{E}$ is the maximum number of edges $i j$, such that $i \notin \operatorname{Participants}(j)$ in all spanning forests of $G$. $C_{E}=\max _{B \in S T(G)} \mid\{i j: i j \in E(B) \wedge i \notin$ Participants $(j)\} \mid$, where $S T(G)$ is the set of all spanning forests of $G$.

- $A_{E}$ is the minimum number of edges to be added in order to get a graph in which each node is connected to its participants.

- $A_{P}$ is the number of contracts that their payments have not been updated yet, this is $\left|\left\{(i, j):(i, j) \in \Gamma, p_{i j} \neq Q(i, j ; G)\right\}\right|$.

Since any sink configuration $(G, \Gamma, \mathbf{P})$ is a pairwise Nash equilibrium, we have that $(G, \Gamma, \mathbf{P})$ is a feasible configuration, $G$ can not contain any cycle, and each node is connected to all its participants.

Lemma 2. If $(G, \Gamma, \mathbf{P})$ is sink configuration then $\Phi(G, \Gamma, \mathbf{P})=\left(0, C_{E}, 0,0\right)$. Furthermore, if $\Phi(G, \Gamma, \mathbf{P})=(0,0,0,0)$ then $(G, \Gamma, \mathbf{P})$ is a sink configuration. 
Proof. If $(G, \Gamma, \mathbf{P})$ is a sink configuration then the graph $G$ is acyclic, $(G, \Gamma, \mathbf{P})$ is a feasible configuration, and all nodes are connected to their participants. Hence $C_{F}=0, A_{P}=0$ and $A_{E}=0$.

If $\Phi(G, \Gamma, \mathbf{P})=(0,0,0,0)$, then the configuration is feasible, $G$ is a spanning forest of $G_{T}$ and for all $u v \in G, u \in \operatorname{Participants}(v)$. Let us show that any action of a node $u$ other than do nothing decrements strictly its utility. Notice that $u$ can not update any of its contracts since the configuration is feasible. Since there are no cycles in $G$ and each $u v \in E$ satisfies that $u \in \operatorname{Participants}(v)$, then $u$ has no incentive to delete a contract (recall that the sink configuration is a proper configuration, an then if $(u, v) \in \Gamma$ then $(v, u) \notin \Gamma)$. If by adding a new contract $(u, v) \in \Gamma$, it does not decrease the utility of $u$, then $p_{u v}<0$. Hence $v$ has no incentive to accept the proposal.

At any round of the Myopic Dynamics neither $C_{E}+A_{E}$ nor $C_{F}$ will be incremented.

Lemma 3. Let $(G, \Gamma, \mathbf{P})$ be any proper configuration and let $u \in V$. Assume that $\Phi(G, \Gamma, \mathbf{P})=\left(C_{F}, C_{E}, A_{E}, A_{P}\right)$ and that $\Phi($ MyopicRound $(u, G, \Gamma, \mathbf{P}))=$ $\left(C_{F}^{\prime}, C_{E}^{\prime}, A_{E}^{\prime}, A_{P}^{\prime}\right)$. Then, for any vertex $u \in V$, we have $C_{E}^{\prime}+A_{E}^{\prime} \leq C_{E}+A_{E}$ and $C_{F}^{\prime} \leq C_{F}$. Moreover, if $A_{E}=0$ then no pair of nodes have incentive to create a new link.

Proof. Let us consider all the possible actions of $u$ after updating the prices of all its contracts:

(i) $u$ does not do anything else. Hence the topology remains the same.

(ii) $u$ breaks a contract $(u, v)$ (or $(v, u))$, then $C_{F}^{\prime} \leq C_{F}$ and $C_{E}^{\prime} \leq C_{E}$. But it might occur that $A_{E}^{\prime}=A_{E}+1$ and then by Assumption 2 we have that $v \notin$ participants $(u)$ and then $C_{E}^{\prime}=C_{E}-1$.

(iii) $u$ asks whether $v$ accepts a contract $(u, v)$ (or $(v, u))$ and $v$ accepts it. Then it can be the case that $C_{u} \neq C_{v}$. If the new link $u v$ connects $u$ or $v$ to one of their (disconnected) participants, then $A_{E}^{\prime}=A_{E}-1$. Otherwise, $u$ only has incentive to add $(u, v)$ if at least $-p_{u v}-\Pi>0$ and $v$ has incentive to accept the contract only if $p_{u v}-\Pi \geq 0$. Since $\Pi>0$, it can not occur. Finally, in the case that $C_{u}=C_{v}$, then $G+u v$ contains a cycle. Notice that we have the same conditions as in the previous case and then the bilateral deviation does not occur. Summarizing, $u v$ does not add a new cycle, $C_{F}^{\prime}=C_{F}, u v$ connects at least two (disconnected) participants, $A_{E}^{\prime}=A_{E}$, and since only a new link is added, $C_{E}^{\prime} \leq C_{E}+1$. Since $u$ can not introduce a new cycle, if $A_{E}=0$ then $u$ has no incentive to add a new link.

However, the previous invariant property of the dynamics is not enough to guarantee convergence. 
Lemma 4. If $\Phi(G, \Gamma, \mathbf{P})=\left(C_{F}, C_{E}, A_{E}, A_{P}\right)$ and $A_{E} \geq 1$, then there exists at least a node $u$ such that its most profitable action is to create a new edge uv where $v \notin C_{u}$ and $v \in$ Participants $(u)$. Then $\Phi\left(G+u v, \Gamma+(u, v), \mathbf{P}^{\prime}\right)=$ $\left(C_{F}, C_{E}, A_{E}-1, A_{P}^{\prime}\right)$.

Proof. Since $A_{E} \geq 1$, there exist $u, v$ such that $u \in \operatorname{Participants}(v)$ and $C_{u} \neq$ $C_{v}$. By Assumptions 1 and 2, $u$ prefers to add a contract to a node of $C_{v}$ instead of cutting any of its contracts. The same occurs with $v$, it prefers to add a contract to a node of $C_{u}$.

Let us suppose that $Q(u, v ; G+u v) \leq 0$, i.e. $v$ pays to $u$. If $u$ is the active node, by the affinity property of $Q$, it is more convenient for $u$ to create a contract with $v$ than with any other $w \notin$ Participants $(u)$. Notice that the traffic through $u$ is the same whatever is the selected node $v^{\prime} \in C_{v}$. Then, the most profitable action for $u$ is to add a contract $\left(u, v^{\prime}\right)$ that maximizes $Q\left(v^{\prime}, u ; G+u v^{\prime}\right)$ considering only nodes $v^{\prime} \in C_{v} \cap$ Participants(u). By Assumptions 1 and $2, v^{\prime}$ accepts the contract $\left(u, v^{\prime}\right)$. If $Q(u, v ; G+u v)>0$, then by the anti-symmetric property $Q(v, u ; G+u v)<0$. Hence we can apply the same argument than above but replacing node active $u$ by node $v$. Then, the most profitable action for $v$ is to add a contract $\left(v, u^{\prime}\right)$ that maximizes $Q\left(u^{\prime}, v ; G+u^{\prime} v\right)$ considering only nodes $u^{\prime} \in C_{u} \cap$ Participants $(v)$. By Assumptions 1 and $2, u^{\prime}$ accepts the contract $\left(v, u^{\prime}\right)$.

Now, we have all the ingredients to prove the convergence of our dynamics. We assume that the activation process is uniform, i.e. for all nodes $u$, $\operatorname{Pr}\left[U_{k}=u\right]=\frac{1}{n}$, where $n$ is the number of nodes ${ }^{1}$.

Theorem 3. The Myopic Dynamics initiated at any initial configuration converges uniformly to a pairwise Nash configuration after $O\left(n^{2} \log n\right)$ rounds in expectation.

Proof. Let $(G, \Gamma, \mathbf{P})$ be any proper configuration and assume that $\Phi(G, \Gamma, \mathbf{P})=$ $\left(C_{F}, C_{E}, A_{E}, A_{P}\right)$. If $A_{E} \geq 1$, then by lemma 5 there exists at least a node $u$ such that its most profitable action is to create a new link with one of its participants without introducing any cycle so that $\Phi\left(G+u v, \Gamma+(u, v), \mathbf{P}^{\prime}\right)=$ $\left(C_{F}, C_{E}, A_{E}-1, A_{P}^{\prime}\right)$. By a coupon collector argument, the expected number of rounds to activate each node at least once is $O(n \log n)$, then $A_{E}$ decreases by one in $O(n \log n)$ rounds in expectation. Hence, after $O\left(n^{2} \log n\right)$ rounds in expectation we have that $A_{E}=0$.

If $A_{E}=0$ then by lemma 4 , no pair of nodes have incentive to create a new link and for any $u \in V$, if $\Phi($ MyopicRound $(u, G, \Gamma, \mathbf{P}))=\left(C_{F}^{\prime}, C_{E}^{\prime}, A_{E}^{\prime}, A_{P}^{\prime}\right)$ then $C_{E}^{\prime}+A_{E}^{\prime} \leq C_{E}+A_{E}$ and $C_{F}^{\prime} \leq C_{F}$. Hence, either $C_{E}^{\prime}=C_{E}-1$ and $A_{E}^{\prime}=1$, or $C_{F}^{\prime}=C_{F}-1$. The worst case is that neither decreases $C_{E}+A_{E}$ nor $C_{F}$. This means that $C_{E}^{\prime}=C_{E}-1$ and $A_{E}^{\prime}=1$ and $C_{F}^{\prime}=C_{F}$. Since $A_{E}^{\prime}=1$,

\footnotetext{
${ }^{1}$ The proof of convergence holds whenever the node activation process guarantees that almost surely all pair of nodes $u$ and $v$ are activated successively infinitely often (however the expected time will not necessarily be the same)
} 
then there exists at least one node that its most profitable action is to create a link with one of its participants and the parameters of the new configuration are $A_{E}^{\prime \prime}=0, C_{E}^{\prime \prime}=C_{E}-1$, and $C_{F}^{\prime \prime}=C_{F}$. Hence in $O(2 n \log n)$ rounds in expectation, $C_{E}$ decreases by one and $A_{E}=0$. Notice that $C_{E} \leq n-1$, then in $O\left(n^{2} \log n\right)$ rounds in expectation the current $C_{E}^{\prime \prime}$ can not be decreased anymore and $C_{F}^{\prime \prime} \geq 0$.

In $O(n \log n)$ additional rounds in expectation all the nodes will be activated once and and then the current $C_{F}$ is decremented at least by one (and by $n$ at most). Then in $O\left(n^{2} \log n\right)$ additional rounds in expectation $C_{F}$ becomes 0 . Finally it only remains to update the payments of all contracts. Hence $A_{p}=0$ in $O(n \log n)$ additional rounds in expectation.

Therefore, the myopic dynamics initiated at any proper and feasible configuration reaches a sink $\left(G^{\prime}, \Gamma^{\prime}, \mathbf{P}_{\text {updated }}\right)$ for which $\Phi\left(\left(G^{\prime}, \Gamma^{\prime}, \mathbf{P}_{\text {updated }}\right)\right)=$ $\left(0, C_{E}^{\prime \prime}, 0,0\right)$ in $O\left(n^{2} \log n\right)$ rounds in expectation.

We notice that the assumptions made on the contracting function are quite natural and, even though the initial configuration and the communication pattern can be any one, the myopic dynamics converges uniformly to a pairwise Nash configuration in a polynomial number of rounds in expectation. Observe that when the traffic matrix represents a uniform all-to-all communication pattern Theorem 3 improves the expected convergence time with respect to the dynamics studied in $[5,7]$.

\section{Conclusions}

In this work we have extended the network formation model of Arcaute et al. $[5,7]$ to nonuniform traffic. Our model considers a traffic matrix for asymmetric players, each pair of nodes can have a different amount of traffic, instead of the uniform all-to-all pattern, in which all traffic demands are equal, considered in $[5,7]$. We have shown a general characterization of the pairwise Nash topologies depending on the traffic. We have focused our study on the definition of an original dynamics model. Our dynamics considers a new strategy space and as long as the assumptions made on the utility function and the contracting function are satisfied, the convergence to a pairwise Nash configuration is guaranteed. Furthermore, under a uniform node activation process we can guarantee convergence in a polynomial number of rounds in expectation. Besides, the expected time $O\left(n^{2} \log n\right)$ of our dynamics improves the time bound $O\left(n^{5}\right)$ obtained, for the dynamics for the uniform all to all traffic, in $[5,7]$, although the assumptions make in both models are not the same.

There are several open research directions. The most obvious is to study the quality of the equilibria with respect to the social optimum. It seems natural to analyze it in relation to the given traffic matrix as well as in relation to the initial configuration in the dynamics. Another interesting direction is the study of other natural requirements on the contracting function that guarantee the 
convergence of the dynamics or showing that the assumptions in this paper are tight.

\section{References}

[1] S. Albers, S. Eilts, E. Even-Dar, Y. Mansour, and L. Roditty. On nash equilibria for a network creation game. In Proceedings of the Seventeenth Annual ACM-SIAM Symposium on Discrete Algorithms, SODA 2006, Miami, Florida, USA, January 22-26, 2006, pages 89-98. ACM Press, 2006.

[2] S. Albers, S. Eilts, E. Even-Dar, Y. Mansour, and L. Roditty. On nash equilibria for a network creation game. ACM Trans. Economics and Comput., $2(1): 2,2014$.

[3] N. Alon, E. D. Demaine, M. Hajiaghayi, P. Kanellopoulos, and T. Leighton. Correction: Basic network creation games. SIAM J. Discrete Math., 28(3):1638-1640, 2014.

[4] N. Alon, E. D. Demaine, M. T. Hajiaghayi, and T. Leighton. Basic network creation games. SIAM J. Discrete Math., 27(2):656-668, 2013.

[5] E. Arcaute, R. Johari, and S. Mannor. Network formation: Bilateral contracting and myopic dynamics. In X. Deng and F. C. Graham, editors, Internet and Network Economics, Third International Workshop, WINE 200\%, San Diego, CA, USA, December 12-14, 200\%, Proceedings, volume 4858 of Lecture Notes in Computer Science, pages 191-207. Springer, 2007.

[6] E. Arcaute, R. Johari, and S. Mannor. Local two-stage myopic dynamics for network formation games. In C. H. Papadimitriou and S. Zhang, editors, Internet and Network Economics, 4th International Workshop, WINE 2008, Shanghai, China, December 17-20, 2008. Proceedings, volume 5385 of Lecture Notes in Computer Science, pages 263-277. Springer, 2008.

[7] E. Arcaute, R. Johari, and S. Mannor. Network formation: Bilateral contracting and myopic dynamics. IEEE Trans. Automat. Contr., 54(8):1765$1778,2009$.

[8] D. Bilò, L. Gualà, and G. Proietti. Bounded-distance network creation games. In P. W. Goldberg, editor, Internet and Network Economics 8th International Workshop, WINE 2012, Liverpool, UK, December 1012, 2012. Proceedings, volume 7695 of Lecture Notes in Computer Science, pages 72-85. Springer, 2012.

[9] U. Brandes, M. Hoefer, and B. Nick. Network creation games with disconnected equilibria. In C. H. Papadimitriou and S. Zhang, editors, Internet and Network Economics, 4th International Workshop, WINE 2008, Shanghai, China, December 17-20, 2008. Proceedings, volume 5385 of Lecture Notes in Computer Science, pages 394-401. Springer, 2008. 
[10] J. Corbo and D. C. Parkes. The price of selfish behavior in bilateral network formation. In M. K. Aguilera and J. Aspnes, editors, Proceedings of the Twenty-Fourth Annual ACM Symposium on Principles of Distributed Computing, PODC 2005, Las Vegas, NV, USA, July 17-20, 2005, pages 99-107. ACM, 2005.

[11] E. D. Demaine, M. Hajiaghayi, H. Mahini, and M. Zadimoghaddam. The price of anarchy in network creation games. In I. Gupta and R. Wattenhofer, editors, Proceedings of the Twenty-Sixth Annual ACM Symposium on Principles of Distributed Computing, PODC 2007, Portland, Oregon, USA, August 12-15, 2007, pages 292-298. ACM, 2007.

[12] E. D. Demaine, M. Hajiaghayi, H. Mahini, and M. Zadimoghaddam. The price of anarchy in cooperative network creation games. In S. Albers and J. Marion, editors, 26th International Symposium on Theoretical Aspects of Computer Science, STACS 2009, February 26-28, 2009, Freiburg, Germany, Proceedings, volume 3 of LIPIcs, pages 301-312. Schloss Dagstuhl Leibniz-Zentrum fuer Informatik, Germany, 2009.

[13] E. D. Demaine, M. T. Hajiaghayi, H. Mahini, and M. Zadimoghaddam. The price of anarchy in cooperative network creation games. SIGecom Exchanges, 8(2):2, 2009.

[14] E. D. Demaine, M. T. Hajiaghayi, H. Mahini, and M. Zadimoghaddam. The price of anarchy in network creation games. ACM Transactions on Algorithms, 8(2):13, 2012.

[15] A. Fabrikant, A. Luthra, E. Maneva, C. H. Papadimitriou, and S. Shenker. On a network creation game. In Proceedings of the Twenty-second Annual Symposium on Principles of Distributed Computing, PODC '03, pages 347351, New York, NY, USA, 2003. ACM.

[16] M. O. Jackson. A survey of models of network formation: Stability and efficiency. Working Papers 1161, California Institute of Technology, Division of the Humanities and Social Sciences, Mar. 2003.

[17] M. O. Jackson and A. Wolinsky. A strategic model of social and economic networks. Journal of Economic Theory, 71:44-74, 1996.

[18] P. Lenzner. On dynamics in basic network creation games. In G. Persiano, editor, Algorithmic Game Theory, 4th International Symposium, SAGT 2011, Amalfi, Italy, October 17-19, 2011. Proceedings, volume 6982 of Lecture Notes in Computer Science, pages 254-265. Springer, 2011.

[19] S. Leonardi and P. Sankowski. Network formation games with local coalitions. In I. Gupta and R. Wattenhofer, editors, Proceedings of the TwentySixth Annual ACM Symposium on Principles of Distributed Computing, PODC 2007, Portland, Oregon, USA, August 12-15, 2007, pages 299-305. ACM, 2007. 